\title{
Procesado electroquímico de un polímero conductor: mimetismo con los metales
}

\author{
S. VILLANUEVA, T.F. OTERO, E. BRILLAS ${ }^{1}$, J. CARRASCO ${ }^{2}$ \\ Laboratorio de Electroquímica. Facultad de Química. Universidad del País Vasco. P.O. Box 1072, 20080 San Sebastián. \\ ${ }^{1}$ Departamento de Química Física. \\ ${ }^{2}$ Departament d'Enginyeria Química i Metal.lúrgia. Facultat de Química. Universitat de Barcelona. Martí i Franquès, 1. 08028 Barcelona.
}

\begin{abstract}
Se ha demostrado que aplicando una densidad de corriente anódica de $0.5 \mathrm{~mA} . \mathrm{cm}^{-2}$ a través de una disolución de acetonitrilo de concentración $5 \mathrm{mM}$ en SNS y $0.1 \mathrm{M}$ en $\mathrm{LiClO}_{4}$, se obtienen películas de poli(SNS) adherentes y uniformes sobre un electrodo de platino. Estas películas poliméricas pueden electrodisolverse (al aplicar una densidad de corriente catódica) y electrodepositarse (al oxidar las cadenas poliméricas que se encuentran reducidas y disueltas en la disolución de fondo). En este trabajo se ha estudiado la influencia de distintos electrolitos: tetraetilamonioperclorato $\left(\mathrm{TEAClO}_{4}\right)$, tetrabutilamonioperclorato $\left(\mathrm{TBAClO}_{4}\right)$ y tetrahexilamonioperclorato $\left(\mathrm{THAClO}_{4}\right)$ en los procesos de electrogeneración, electrodisolución y electrodepósito.
\end{abstract}

Palabras clave: Electrogeneración, electrodisolución, electrodepósito, polímeros conductores solubles.

Electrochemical processability of a conducting polymer: mimicking inorganic metals

When an anodic current density of $0.5 \mathrm{~mA} . \mathrm{cm}^{-2}$ is applied during $60 \mathrm{~s}$ through acetonitrile solutions containing, $5 \mathrm{mM} \mathrm{SNS}$ $+0.1 \mathrm{M} \mathrm{LiClO}_{4}$, adherent and uniform poly(SNS) films are electrogenerated on a platinum electrode. These polymeric films can be electrodissolved in the background solution when a cathodic current density is applied. They can be also electrodeposited from solutions of the reduced polymer in the background solution by flow of an anodic current. In this work the influence of different electrolytes: tetraethylammonium perchlorate $\left(\mathrm{TEAClO}_{4}\right)$, tetrabutylammonium perchlorate $\left(\mathrm{TBAClO}_{4}\right)$ and tetrahexyl-ammonium perchlorate $\left(\mathrm{THAClO}_{4}\right)$ in electrogeneration, electrodissolution and electro deposition processes has been studied.

Keywords: Electrogeneration, electrodissolution, electrodeposition, soluble conducting polymers.

\section{INTRODUCCIÓN}

Los polímeros conductores son materiales que tienen propiedades electrocrómicas (1), electroquimiomecánicas (2) y conductoras de la electricidad (3). Sin embargo, el desarrollo de éstas y otras propiedades se ha visto limitado por la baja estabilidad y la baja procesabilidad de estos materiales. En los últimos años se ha realizado un gran esfuerzo con el objeto de superar ambas limitaciones tecnológicas. La baja procesabilidad debida a la insolubilidad o infusibilidad de los polímeros conductores se ha mejorado con la síntesis de oligómeros solubles a partir de monómeros sustituidos con cadenas laterales, generando una secuencia en bloque de copolímeros (poliheterociclos) $(4,5)$.

Un caso especial de copolímeros en bloque es la generación química de dímeros y trímeros que poseen dos o más anillos heteroaromáticos derivados del tiofeno, furano y pirrol. En este camino, nuestro grupo ha mejorado las condiciones de síntesis de diferentes trímeros, tales como 2,5-di-(-2-tienil) pirrol (SNS) (6) y 2,5-di-(-2-tienil)tiofeno (SSS) $(7,8)$.

La baja solubilidad de las películas oxidadas de poli(SNS) en acetonitrilo cae a cero cuando diferentes sales se disuelven en dicho disolvente. Sin embargo la forma reducida de la película es muy soluble en este medio. Por lo tanto, las películas de poli(SNS) electrogeneradas pueden disolverse cuantitativamente al aplicar una densidad de corriente catódica en la disolución de fondo, denominándose este proceso electrodisolución.
El objeto de este trabajo es comprobar si los oligómeros electrodisueltos pueden ser depositados de nuevo desde la disolución por oxidación electroquímica, dando lugar a una completa procesabilidad electroquímica del material polimérico. Este proceso se denomina electrodepósito y se ha estudiado utilizando distintos electrolitos.

Se puede hablar, por vez primera, de un "material orgánico" susceptible de ser electrodepositado por oxidación y electrodisuelto por reducción, procesos que, de hecho son contrarios a los de los metales que se electrodepositan por reducción de sus iones y se electrodisuelven al oxidarse.

\section{PROCEDIMIENTO EXPERIMENTAL}

El monómero SNS fue sintetizado como se ha indicado previamente (6). El acetonitrilo de la casa Lab Scan y de grado HPLC fue utilizado como disolvente. Las sales de amonio empleadas: tetraetilamonio perclorato $\left(\mathrm{TEAClO}_{4}\right)$, tetrabutilamonio perclorato $\left(\mathrm{TBAClO}_{4}\right)$ y tetrahexilamonio perclorato $\left(\mathrm{THAClO}_{4}\right)$ fueron de la casa Fluka y de grado puro, mientras que el perclorato de litio usado fue de la casa Aldrich y de grado A. C. S. Estas sales se secaron en un horno a una temperatura de $80^{\circ} \mathrm{C}$ antes de ser empleadas.

Todos los estudios electroquímicos fueron realizados en una 
celda de tres orificios de un único compartimento y termostatizada. Se emplearon como electrodo de trabajo y contraelectrodo dos láminas de platino de 1 y $4 \mathrm{~cm}^{2}$ cada una. Ambos electrodos se limpiaron primeramente por inmersión en mezcla sulfocrómica, fueron aclarados en agua destilada y por último en acetonitrilo antes de empezar cada electropolimerización. Las medidas cronoamperométricas y cronopotenciométricas fueron realizadas usando un potenciostato-galvanostato PAR 273A, el cual está conectado a un ordenador personal a través del programa PAR M270.

Antes de ser empleada cada disolución fue desoxigenada borboteando nitrógeno, previamente presaturado en acetonitrilo, durante 15 minutos.

Una vez electropolimerizadas las películas se secan en aire caliente, se enjuagan varias veces en acetonitrilo, se secan de nuevo y se pesan hasta obtener un peso constante. El peso de los electrodos y de las películas poliméricas fue determinado con una ultramicrobalanza Sartorious 4504 MPS que tiene una precisión de $10^{-7} \mathrm{~g}$.

\section{RESULTADOS Y DISCUSIÓN}

\subsection{Electropolimerización}

Cuando se aplica una densidad de corriente anódica a través de una disolución que contiene el disolvente, acetonitrilo, la sal, $\mathrm{LiClO}_{4}$ y el monómero, SNS, tiene lugar la formación de una película polimérica, poli(SNS), sobre el electrodo de platino. Este proceso se denomina electropolimerización o electrogeneración. El polímero se forma en estado oxidado. Esta película polimérica es soluble en algunos disolventes orgánicos, DMSO, metanol y acetona, parcialmente soluble en acetonitrilo e insoluble en disoluciones acuosas (9). Sin embargo su solubilidad en acetonitrilo cae a cero cuando se disuelve en él una sal como el $\mathrm{LiClO}_{4}$.

En trabajos anteriores $(6,10,11)$ se ha observado que la electrogeneración es un proceso faradaico: la masa de polímero electrogenerada es proporcional a la carga consumida, siendo el valor de la productividad, masa de polímero que se genera sobre el electrodo por cada miliculombio que se consume, en estas condiciones de $2.1 \times 10^{-3} \mathrm{mg}$. $\mathrm{mC}^{-1}$. En este trabajo se ha estudiado la influencia que tienen las distintas sales de amonio (anteriormente citadas) en el proceso de electrogeneración (tabla I).

Como podemos observar en la tabla I, a medida que aumenta el tamaño del catión que se utiliza durante la electrogeneración, la masa de polímero que se genera sobre el electrodo de $\mathrm{Pt}$ disminuye. Las productividades obtenidas tienen un valor de: $1.6 \times 10^{-3}, 1.3 \times 10^{-3}$ y $1.1 \times 10^{-3} \mathrm{mg} . \mathrm{mC}^{-1}$ para $\mathrm{TEAClO}_{4}$ $\mathrm{TBAClO}_{4}$ y $\mathrm{THAClO}_{4}$ respectivamente.

\subsection{Proceso de electrodisolución.}

Es el proceso por el cual una película electropolimerizada se disuelve en una disolución electrolítica al realizar una reducción electroquímica.

Cuando se somete a las películas de poli(SNS) electrogeneradas a potenciales más negativos que $0.2 \mathrm{~V}$, aparece una nube amarilla alrededor del electrodo. Tiene entonces lugar el proceso de electrodisolución. Al final de la disolución electroquímica se observa un salto de potencial a valores más negativos. Sin embargo cuando se introduce el electrodo recubierto de poli(SNS) en una disolución de acetonitrilo de concentración $0.1 \mathrm{M}^{\text {en }} \mathrm{LiClO}_{4}$ y se polariza a potenciales superiores a $0.4 \mathrm{~V}$,
TABLA I. INFLUENCIA DE LAS SALES DE AMONIO EN LA ELECTROGENERACIÓN DE LAS PELÍCULAS DE POLI(SNS).

\begin{tabular}{|c|c|c|c|}
\hline & \multicolumn{3}{|c|}{ Masa electrogenerada/ mg } \\
\hline Tiempo/ s & TEAClO $_{4}$ & TBAClO $_{4}$ & THAClO $_{4}$ \\
\hline 10 & 0.0084 & 0.0070 & 0.0055 \\
\hline 20 & 0.0182 & 0.0137 & 0.0096 \\
\hline 30 & 0.0262 & 0.0200 & 0.0167 \\
\hline 40 & 0.0344 & 0.0264 & 0.0227 \\
\hline 50 & 0.0414 & 0.0320 & 0.0274 \\
\hline 60 & 0.0490 & 0.0393 & 0.0327 \\
\hline
\end{tabular}

TABLA II. INFLUENCIA DE LAS SALES DE AMONIO EN LA ELECTRODISOLUCIÓN DE LAS PELÍCULAS DE POLI(SNS).

\begin{tabular}{|l|l|c|l|}
\hline & \multicolumn{3}{|c|}{ Masa electrodisuelta/ mg } \\
\hline Tiempo/s & TEAClO $_{4}$ & TBAClO $_{4}$ & THAClO $_{4}$ \\
\hline 4 & 0.0050 & 0.0032 & 0.0023 \\
\hline 8 & 0.0097 & 0.0104 & 0.0071 \\
\hline 12 & 0.0147 & 0.0237 & 0.0137 \\
\hline 16 & 0.0191 & 0.0302 & 0.0172 \\
\hline 20 & 0.0250 & 0.0357 & 0.0224 \\
\hline 24 & 0.0305 & 0.0380 & 0.0257 \\
\hline
\end{tabular}

no se produce la electrodisolución de las películas permaneciendo éstas insolubles en dichas condiciones.

En trabajos previos se ha realizado un seguimiento microgravimétrico del proceso de electrodisolución $(6,10,11)$. Para ello se han electrogenerado películas de misma masa a partir de disoluciones monoméricas que contienen $\mathrm{LiClO}_{4}$ como electrolito y se les ha aplicado una densidad de corriente catódica de $0.2 \mathrm{~mA} . \mathrm{cm}^{-2}$ durante distintos tiempos. A partir de los resultados obtenidos se puede decir que el proceso de electrodisolución es un proceso faradaico, ya que la masa que se electrodisuelve es proporcional a la carga catódica que se inyecta. La productividad del proceso de electrodisolución en estas condiciones es de $7.2 \times 10^{-3} \mathrm{mg}$. $\mathrm{mC}^{-1}$. Es decir por cada $\mathrm{mC}$ de carga catódica que se inyecta se electrodisuelven $7.2 \times 10^{-3} \mathrm{mg}$ de polímero.

Al igual que en el apartado anterior se ha estudiado la influencia de las distintas sales de amonio en la electrodisolución. Para ello se han electrogenerado películas de poli(SNS) a partir de disoluciones de acetonitrilo de concentración $5 \mathrm{mM}$ en SNS y $0.1 \mathrm{M}$ en las distintas sales, aplicando a través de ellas una densidad de corriente anódica de $0.5 \mathrm{~mA} . \mathrm{cm}^{-2}$ durante 60 s. Una vez electrogeneradas las películas de poli(SNS) se han llevado cada una a su disolución de fondo (disolución inicial sin monómero) y se les ha aplicado una densidad de corriente catódica de $0.2 \mathrm{~mA} . \mathrm{cm}^{-2}$ durante distintos tiempos. Los resultados obtenidos se muestran en la tabla II.

La productividad del proceso de electrodisolución para las distintas sales tiene un valor de: $6.4 \times 10^{-3}, 5.7 \times 10^{-3}$ y $7.4 \times 10^{-3} \mathrm{mg}$. $\mathrm{mC}^{-1}$ para $\mathrm{TEAClO}_{4}, \mathrm{TBAClO}_{4}$ y $\mathrm{THAClO}_{4}$ respectivamente.

La productividad del proceso de electrodisolución, que indica la masa de polímero que se disuelve por cada carga catódica consumida es, en todos los casos, mucho mayor que la productividad del proceso de electropolimerización. Esto es debido a que durante la polimerización la carga se consume en dos pro- 
cesos simultáneos: oxidación del monómero [1] y del polímero [2]. Sin embargo durante la electrodisolución la carga sólo se consume en la reducción del polímero [3]. La electropolimerización y la electrodisolución no son procesos reversibles.

$$
\begin{aligned}
& {\left[(\mathrm{SNS})_{\mathrm{n}}^{+}\right]_{\mathrm{s}}+\left[(\mathrm{SNS})^{\dot{+}}\right]_{\mathrm{sol}} \longrightarrow\left[(\mathrm{SNS})_{\mathrm{n}+1}\right]_{\mathrm{s}}+2 \mathrm{H}^{+}{ }_{[\text {Polimerización }]}[1]} \\
& \left.[(\mathrm{SNS}) \mathrm{n}]_{\mathrm{S}}+\mathrm{a}\left(\mathrm{ClO}_{4}^{-}\right)_{\mathrm{sol}} \longrightarrow\left[\left(\mathrm{SNS}^{\mathrm{a}+}\right)_{\mathrm{n}}\left(\mathrm{ClO}_{4}^{-}\right)_{\mathrm{an}}\right]_{\mathrm{S}}+\mathrm{a}^{\left(\mathrm{e}^{-}\right)}\right)_{\mathrm{me}}{ }^{\text {[Dopado-oxidación] [2] }} \\
& \mathrm{n}\left(\mathrm{e}^{-}\right) \mathrm{me}+\left[\left(\mathrm{SNS}_{\mathrm{n}^{\mathrm{a}}}{ }^{\mathrm{a}}\left(\mathrm{ClO}_{4}\right)^{-}\right) \mathrm{a}\right] \mathrm{s} \longrightarrow\left[(\mathrm{SNS})_{\mathrm{n}}\right]_{\mathrm{sol}}+\mathrm{a}\left(\mathrm{ClO}_{4}^{-}\right) \text {[Electrodisolución] [3] }
\end{aligned}
$$

donde el subíndice "sol" significa disolución, el subíndice "s" se refiere a la película sólida y "me" al electrodo metálico.

\subsection{Proceso de electrodepósito.}

Es el proceso en el que tiene lugar la oxidación de las moléculas poliméricas neutras, disueltas en la disolución electrolítica, para dar lugar a una película polimérica oxidada e insoluble en dicha disolución. Esta película mantiene su solubilidad en disolventes orgánicos y puede ser electrodisuelta.

Para estudiar el proceso de electrodepósito es necesario preparar disoluciones de acetonitrilo que contengan polímero reducido. Para ello se polimerizan películas de poli(SNS) a partir de disoluciones monoméricas y se electrodisuelven, en una celda distinta a la anterior, hasta obtener la cantidad de polímero reducido y disuelto deseada. Una vez alcanzada esta concentración se aplica una intensidad de corriente anódica. Tiene lugar el proceso de electrodepósito de las películas de poli(SNS). Tenemos entre manos un nuevo proceso faradaico ya que, al igual que en los casos anteriores, la masa de polímero depositada aumenta con la carga consumida (12).

Las películas se obtienen mediante cronopotenciometría (intensidad de corriente constante) y no mediante cronoamperometría (potencial constante) ya que en estas condiciones las películas de poli(SNS) que se obtienen sobre el electrodo no son adherentes.

La mejor productividad del proceso de electrodepósito, $1.4 \mathrm{x}$ $10^{-3} \mathrm{mg}$. $\mathrm{mC}^{-1}$, empleando $\mathrm{LiClO}_{4}$ como electrolito, fue obtenida al aplicar una densidad de corriente de $8 \mu \mathrm{A} . \mathrm{cm}^{-2}$ a través de disoluciones de acetonitrilo de concentración $0.1 \mathrm{M}$ en el electrolito y en las que previamente se había reducido y disuelto $0.75 \mathrm{mg}$ de poli(SNS) (13), en $6 \mathrm{ml}$. de disolución.

Observando la productividad del proceso de electrodepósito, $1.4 \times 10^{-3} \mathrm{mg}$. $\mathrm{mC}^{-1}$, con la de electrodisolución, $7.2 \times 10^{-3}$ mg. $\mathrm{mC}^{-1}$, vemos que ambos procesos no son reversibles, debido a que durante el depósito, en estas condiciones, tienen lugar reacciones paralelas que consumen parte de la carga que se aplica sin que se deposite polímero (13). Para disminuir la influencia de estas reacciones, se trabajó a temperaturas inferiores $\left(2^{\circ} \mathrm{C}\right)$ obteniendo así un mayor depósito de polímero sobre el electrodo, $3.3 \times 10^{-3} \mathrm{mg}$. $\mathrm{mC}^{-1}$.

Una vez obtenidas las películas de poli(SNS) depositadas, éstas se electrodisuelven hasta un $70 \%$ de su peso al aplicar sobre ellas en la disolución de fondo un potencial negativo de $0.4 \mathrm{~V}$. Las películas depositadas son capaces de solubilizarse también en DMSO. Al ser controladas electroquímicamente siguen manteniendo su capacidad electrocrómica adquiriendo un color azul intenso al ser oxidadas y amarillo-anaranjado al ser reducidas.

El hecho de que estas películas no pueden electrodisolverse totalmente es debido a que durante el proceso de electrodepó-

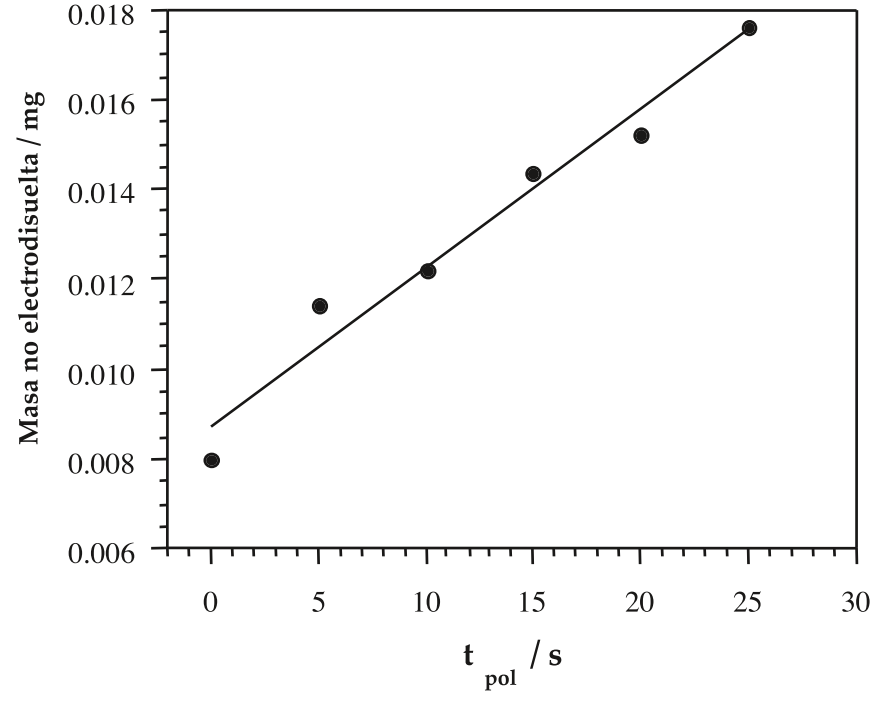

Figura 1. Variación de la masa de poli(SNS) que queda sobre el electrodo de platino con el tiempo de polarización. Se generaron películas de poli(SNS) de misma masa al aplicar una densidad de corriente de $0.5 \mathrm{~mA} . \mathrm{cm}^{-2}$ durante $60 \mathrm{~s}$ a través de una disolución $5 \mathrm{mM}$ en SNS y $0.1 \mathrm{M}$ en $\mathrm{TEAClO}_{4}$. Una vez generadas fueron polarizadas en la disolución de fondo (sin monómero) a un potencial de $750 \mathrm{mV}$ durante distintos tiempos (una película nueva cada vez). Entonces fueron sometidas a una corriente catódica de $0.2 \mathrm{~mA} . \mathrm{cm}^{-2}$ en la disolución de fondo disolviéndose parcialmente. Mediante una microbalanza y por diferencia de pesada de las películas secas, se determinó la cantidad de polímero disuelta a cada tiempo.

sito las películas sufren procesos de reticulación. Estos procesos se hacen más patentes durante el electrodepósito que durante la electrogeneración o electrodisolución. Esto es debido a que el proceso de electrodepósito tiene lugar a tiempos largos, 1800 s mientras que la electrogeneración y la electrodisolución tienen lugar a tiempos más cortos (60 y 30 s respectivamente).

\subsection{Proceso de reticulación}

Para estudiar los procesos de reticulación se electrogeneraron películas de poli(SNS) de misma masa aplicando una densidad de corriente anódica de $0.5 \mathrm{~mA} . \mathrm{cm}^{-2}$ durante $60 \mathrm{~s}$ a través de una disolución de acetonitrilo de concentración 5

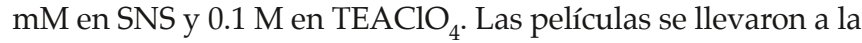
disolución de fondo donde se les aplicó un potencial de 550 $\mathrm{mV}$ durante diferentes tiempos. Una vez polarizadas se controlaron por voltamperometría cíclica en la misma disolución de fondo. Durante el control las películas se electrodisolvieron. Para determinar la cantidad de masa de poli(SNS) que se electrodisolvió en cada experiencia se pesó el electrodo de platino recubierto de polímero antes y después de ser controlado.

Los resultados obtenidos se muestran en la figura 1. En esta gráfica se observa cómo varía la cantidad de polímero que queda sobre el electrodo con el tiempo de polarización. Así la masa de polímero que queda reticulada aumenta con el tiempo de reticulación. 


\section{CONCLUSIONES}

Tenemos un monómero, SNS, a partir del cual y mediante electropolimerización va a dar lugar a un polímero, poli(SNS), siguiendo un proceso faradaico. La productividad de este proceso varía en función del electrolito empleado, disminuyendo a medida que aumenta el tamaño de la sal: $\mathrm{LiClO}_{4}, \mathrm{TEAClO}_{4}$ $\mathrm{TBAClO}_{4}$ y $\mathrm{THAClO}_{4}$. Si se aplica una densidad de corriente catódica a través de estas películas, éstas se reducen y se disuelven teniendo lugar el proceso de electrodisolución. Éste es también un proceso faradaico. Cuando se aplica una densidad de corriente anódica a través de disoluciones que contienen cadenas de polímero reducidas, éstas se oxidan depositándose de nuevo sobre el electrodo de platino. Este proceso se denomina electrodepósito y se ha observado que es un proceso faradaico. Cuando el electrodepósito tiene lugar a temperaturas bajas, su productividad se aproxima más a la del proceso de electrodisolución. Durante el depósito las películas sufren un proceso de reticulación que aumenta a medida que aumenta el tiempo de electrodepósito. La reticulación de las películas electrogeneradas y electrodepositadas puede ser controlada electroquímicamente.

\section{AGRADECIMIENTOS}

Los autores desean expresar su agradecimiento al M.E.C., a la Diputación Foral de Guipúzcoa y a la Universidad del País Vasco por el apoyo económico prestado.

\section{BIBLIOGRAFIA}

1. T.F Otero \& M. Bengoechea, "UV-Visible Spectroelectroche-mistry of conducting polymers. Energy linked to conformational changes". Langmuir, (in press).

2. J.M. Sansiñena, V. Olazábal, T.F. Otero, C.N. Polo Da Fonseca \& M.A. De Paoli, "A solid state artificial muscle based on polypyrrole and a solid polymeric electrolyte working in air". Chem. Commun., 2217 - 2218 (1997).

3. T.F. Otero, "Conducting polymers, electrochemistry, and biomimicking processes" in Modern Aspects of Electrochemistry (Ed. by White R.E.) 33, 307-434 (Kluwer Academic, New York, 1999).

4. G. G. Mcleod, M.G.B. Mahboubian-Jones, R.A. Pethrick, S. D. Watson, N.D. Truog, J.C. Galin, J. François, “Synthesis, electrochemical polymerization and properties of poly(2,5-di-(-2-thienyl)pyrrole)". Polymer 27, 455 - 458 (1986).

5. J. P. Ferraris, G.D. Skiles, "Substitutional alloys of organic polymeric conductors". Polymer 28, 179 - 182 (1987).

6. J. Carrasco, A. Figueras, T.F. Otero \& E. Brillas, "Anodic electrosynthesis and cathodic electrodissolution of poly(2,5-di-(2-thienyl)pyrrole. A new way of processability". Synth. Met., 61, 253 - 258 (1993).

7. J. Carrasco, T.F. Otero, E. Brillas, M. Montilla, “Electrogeneration and solubilities of oxidized poly(2,5-di-(-2-thienyl)thiophene". J. Electroanal. Chem. , 418, 115122, (1996).

8. E. Brillas, M. Montilla, J. Carrasco, T.F. Otero, “Electrochemical of poly(2,5-di-(2-thienyl)-thiophene". J. Electroanal. Chem., 418, 123-130, (1996).

9. E. Brillas, J. Carrasco, A. Figueras, F. Urpí \& T.F. Otero, “Oxidized and reduced poly(2,5-di-(-2-thienyl)pyrrole): solubilities, electrodissolution and molar mass". J. Electroanal. Chem., 392, 55-61 (1995).

10. T.F Otero, J. Carrasco, A. Figueras \& E. Brillas, "Electrochemical oxidation of 2,5-di-(-2-thienyl)pyrrole in acetonitrile: cathodic stripping of the electrogenerated conducting polymer". J. of Electroanal. Chem., 370, 231 - 239 (1994).

11. T.F. Otero, E. Brillas, A. Figueras, "Poly(SNS) quantitative electrosynthesis and electrodissolution". Mat. Res. Soc. Symp. Proc., 328, 799-804 (1994)

12. T.F. Otero, S. Villanueva, M. Bengoechea, E. Brillas \& J. Carrasco, "Reversible redox switching in polypyrrole and poly(SNS) films". Synth. Met., 84, 183 - 184 (1997).

13. T.F. Otero, S. Villanueva, E. Brillas \& J. Carrasco, “Electrochemical processing of the conducting polymer poly(SNS)". Acta Polym., 49, 433 - 438 (1998). 